\title{
Existence of positive solutions for integral boundary value problems of fractional differential equations with $p$-Laplacian
}

\author{
Luchao Zhang ${ }^{1,2}$, Weiguo Zhang ${ }^{2 *}$, Xiping Liü and Mei Jia ${ }^{2}$
}

\section{${ }^{*}$ Correspondence:}

zwgzwm@126.com

${ }^{2}$ College of Science, University of

Shanghai for Science and

Technology, Shanghai, 200093,

P.R. China

Full list of author information is

available at the end of the article

\begin{abstract}
This paper is concerned with the existence of positive solutions for integral boundary value problems of Caputo fractional differential equations with $p$-Laplacian operator. By means of the properties of the Green's function, Avery-Peterson fixed point theorems, we establish conditions ensuring the existence of positive solutions for the problem. As an application, an example is given to demonstrate the main result.
\end{abstract}

Keywords: fractional differential equations; Caputo derivative; $p$-Laplacian operator; integral boundary conditions; positive solutions

\section{Introduction}

Recently, fractional differential equations have been proved to be valuable tools in the modeling of many phenomena in various fields of science and engineering, such as rheology, dynamical processes in self-similar and porous structures, heat conduction, control theory, electroanalytical chemistry, chemical physics, economics, etc. Many researchers have shown their interest in fractional differential equations. The motivation for this work stems from both the intensive development of the theory of fractional calculus itself and the applications. Many papers and books have appeared on fractional calculus and fractional differential equations (see [1-10]).

It is well known that the $p$-Laplacian operator is also used in analyzing mechanics, physics, and dynamic systems, and the related fields of mathematical modeling. However, there are few studies of the existence of positive solution of fractional differential equations with the $p$-Laplacian operator; see [11-18] and the references therein.

In [11], Liu et al. studied the solvability of the Caputo fractional differential equation with boundary value conditions involving the $p$-Laplacian operator. The existence and uniqueness of the problem is found by the Banach fixed point theorem. The problem is given in the following:

$$
\left\{\begin{array}{l}
\left(\varphi_{p}\left(D_{0+}^{\alpha} x(t)\right)\right)^{\prime}=f(t, x(t)), \quad \text { for } t \in(0,1) \\
x(0)=r_{0} x(1), \\
x^{\prime}(0)=r_{1} x^{\prime}(1) \\
x^{(j)}(0)=0
\end{array}\right.
$$

(c) The Author(s) 2017. This article is distributed under the terms of the Creative Commons Attribution 4.0 International License (http://creativecommons.org/licenses/by/4.0/), which permits unrestricted use, distribution, and reproduction in any medium, provided you give appropriate credit to the original author(s) and the source, provide a link to the Creative Commons license, and indicate if changes were made. 
where $i=2,3, \ldots,[\alpha]-1$. Here, $\varphi_{p}$ is the $p$-Laplacian operator and $D_{0^{+}}^{\alpha}$ is the Caputo fractional derivative, $1<\alpha \in \mathbb{R}$, and the nonlinear function $f \in C([0,1] \times \mathbb{R}, \mathbb{R})$ is given.

In [12], Lu et al. studied the existence of non-negative solutions of a nonlinear fractional boundary value problem with the $p$-Laplacian operator

$$
\left\{\begin{array}{l}
D_{0+}^{\beta}\left(\varphi_{p}\left(D_{0+}^{\alpha} u(t)\right)\right)=f(t, u(t)), \quad \text { for } 0<t<1, \\
u(0)=u^{\prime}(0)=u^{\prime}(1)=0 \\
D_{0+}^{\alpha} u(0)=D_{0+}^{\alpha} u(1)=0
\end{array}\right.
$$

where $2<\alpha \leq 3,1<\beta \leq 3$, and $D_{0+}^{\alpha}, D_{0+}^{\beta}$ are the standard Riemann-Liouville fractional derivatives. Green's functions, the Guo-Krasnoselskii theorem, and the Leggett-Williams fixed point theorems are used.

Boundary value problems with integral boundary conditions for ordinary differential equations represent a very interesting and important class of problems. They include two-point, three-point and multi-point boundary value problems as special cases. For an overview of the literature on integral boundary value problems and symmetric solutions, see [19-26] and the references therein.

In [24], Zhi et al. studied the existence of positive solutions for nonlocal boundary value problem of the fractional differential equations with $p$-Laplacian operator. The problem is given in the following:

$$
\left\{\begin{array}{l}
\left(\varphi_{p}\left(D_{0+}^{\alpha} u(t)\right)\right)^{\prime \prime}=f\left(t, u(t), D_{0+}^{\beta} u(t)\right), \quad \text { for } 0<t<1, \\
u(0)=u^{\prime \prime}(0)=0, \quad u(1)=\int_{0}^{1} g(s) u(s) d s, \\
\left(\varphi_{p}\left(D_{0+}^{\alpha} u(0)\right)\right)^{\prime}=\lambda_{1}\left(\varphi_{p}\left(D_{0+}^{\alpha} u\left(\xi_{1}\right)\right)\right)^{\prime}, \\
\varphi_{p}\left(D_{0+}^{\alpha} u(1)\right)=\lambda_{2}\left(\varphi_{p}\left(D_{0+}^{\alpha} u\left(\xi_{2}\right)\right)\right),
\end{array}\right.
$$

where $0<\xi_{1} \leq \xi_{2}<1,2<\alpha<3,1<\beta<\alpha-1<2,0 \leq \lambda_{1}, \lambda_{2}<1$. $D_{0+}^{\alpha}$ is the Caputo fractional derivative of order $\alpha$.

In [26], Mahmudov and Unul studied the existence of the solutions of the fractional differential equation with $p$-Laplacian operator and integral conditions is discussed. The problem is given in the following:

$$
\left\{\begin{array}{l}
D_{0+}^{\beta} \phi_{p}\left(D_{0+}^{\alpha} u(t)\right)=f\left(t, u(t), D_{0+}^{\gamma} u(t)\right) \\
u(0)+\mu_{1} u(1)=\sigma_{1} \int_{0}^{1} g(s, u(s)) d s \\
u^{\prime}(0)+\mu_{2} u^{\prime}(1)=\sigma_{2} \int_{0}^{1} h(s, u(s)) d s \\
D_{0+}^{\alpha} u(0)=0 \\
D_{0+}^{\alpha} u(1)=v D_{0+}^{\alpha} u(\eta)
\end{array}\right.
$$

where $D_{0+}^{\alpha}, D_{0+}^{\beta}$ are the Caputo fractional derivative operators with $1<\alpha<2,1<\beta<2 . \mu_{1}$, $\sigma_{i}(i=1,2)$ are non-negative constants. $f, g, h$ are continuous functions.

Motivated by the above work, we investigate the following integral boundary value problems (for short, BVP) of fractional differential equations with $p$-Laplacian:

$$
\left\{\begin{array}{l}
D_{0+}^{\beta} \varphi_{p}\left(D_{0+}^{\alpha} x(t)\right)=f\left(t, x(t), D_{0+}^{\beta} x(t)\right), \quad t \in(0,1) \\
\left(\varphi_{p}\left(D_{0+}^{\alpha} x(0)\right)\right)^{(i)}=\varphi_{p}\left(D_{0+}^{\alpha} x(1)\right)=0, \quad i=1,2, \ldots, m-1, \\
x(1)=\int_{0}^{1} g_{1}(s) x(s) d s, \\
x^{\prime}(0)=\int_{0}^{1} g_{2}(s) x(s) d s, \\
x^{(j)}(0)=0, \quad j=2,3, \ldots, n-1,
\end{array}\right.
$$


where $\varphi_{p}$ is the $p$-Laplacian operator, $1<n-1<\alpha<n, 1<m-1<\beta<m, \alpha-\beta>1$, and $D_{0^{+}}^{\alpha}$ and $D_{0^{+}}^{\beta}$ are the Caputo fractional derivatives. $g_{k} \in C([0,1],[0,+\infty)), k=1,2, f \in C([0,1] \times$ $[0,+\infty) \times(-\infty, 0],[0,+\infty))$ are given functions.

In this paper, a positive solution $x=x(t)$ of BVP (1.1) means a solution of (1.1) satisfying $x(t)>0, t \in[0,1]$.

Throughout this paper, we always assume that the following condition is satisfied.

$\left(\mathrm{L}_{0}\right) g_{1}(t)>g_{2}(t) \geq 0,0 \leq \int_{0}^{1} g_{2}(s) d s, \int_{0}^{1} g_{1}(s) d s<1$.

The organization of the paper is as follows. In Section 2, we present some necessary definitions and lemmas which will be used to prove our main results. In Section 3, by using the Avery-Peterson fixed point theorems, the results for the existence of multiple positive solutions of BVP (1.1) are established. In Section 4, we give an example to demonstrate the main result.

\section{Preliminaries and lemmas}

In this section, we give some definitions and basic lemmas that will be used and important to us in the following.

Definition 2.1 (see [1]) The Riemann-Liouville integral of fractional order $\alpha>0$ of a function $g$ is defined as

$$
I_{0^{+}}^{\alpha} g(t):=\frac{1}{\Gamma(\alpha)} \int_{0}^{t}(t-s)^{\alpha-1} g(s) d s
$$

Definition 2.2 (see [2]) The Caputo derivative of fractional order $\alpha>0$ of a function $g$ is defined as

$$
D_{0+}^{\alpha} g(t)=\frac{1}{\Gamma(n-\alpha)} \int_{0}^{t}(t-s)^{n-\alpha-1} g^{(n)}(s) d s
$$

where $n$ is the smallest integer greater than or equal to $\alpha$.

Definition 2.3 (see [4]) Let $E$ be a real Banach space. A nonempty, closed, and convex set $P \subset E$ is a cone if the following two conditions are satisfied:

(1) if $x \in P$ and $\mu \geq 0$, then $\mu x \in P$;

(2) if $x \in P$ and $-x \in P$, then $x=0$.

Every cone $P \subset E$ induces the ordering in $E$ given by $x_{1} \leq x_{2}$ if and only if $x_{2}-x_{1} \in P$.

Definition 2.4 (see [4]) The map $\gamma$ is said to be a continuous non-negative convex (concave) function on a cone $P$ of a real Banach space $E$ provided that $\gamma: P \rightarrow[0,+\infty)$ is continuous and

$$
\gamma(t x+(1-t) y) \leq(\geq) t \gamma(x)+(1-t) \gamma(y), \quad x, y \in P, t \in[0,1] .
$$

Lemma 2.1 (see [8]) Let $\alpha>0$, assume that $u, D_{0^{+}}^{\alpha} u \in C(0,1) \cap L^{1}(0,1)$, then

$$
I_{0+}^{\alpha} D_{0+}^{\alpha} u(t)=u(t)+C_{0}+C_{1} t+C_{2} t^{2}+\cdots+C_{n-1} t^{n-1}
$$


holds for some $C_{i} \in \mathbb{R}, i=0,1,2, \ldots, n-1$, where $n$ is the smallest integer greater than or equal to $\alpha$.

Lemma 2.2 (see [26]) The Caputo fractional derivative of order $n-1<\alpha<n$ for $t^{\beta}$ is given by

$$
D_{0+}^{\alpha} t^{\beta}= \begin{cases}\frac{\Gamma(\beta+1)}{\Gamma(\beta-\alpha+1)} t^{\beta-\alpha}, & \beta>n-1, \\ 0, & \beta \in\{0,1, \ldots, n-1\} .\end{cases}
$$

Lemma 2.3 Let $h \in C[0,1]$ and $1<m-1<\beta<m$. Then the $B V P$

$$
\left\{\begin{array}{l}
D_{0^{+}}^{\beta} u(t)=h(t), \quad 0<t<1, \\
u(1)=u^{(i)}(0)=0, \quad i=1,2, \ldots, m-1,
\end{array}\right.
$$

has an unique solution

$$
u(t)=-\int_{0}^{1} H(t, s) h(s) d s,
$$

where

$$
H(t, s)=\frac{1}{\Gamma(\beta)} \begin{cases}(1-s)^{\beta-1}-(t-s)^{\beta-1}, & 0 \leq s \leq t \leq 1 \\ (1-s)^{\beta-1}, & 0 \leq t \leq s \leq 1\end{cases}
$$

Proof From (2.1) and Lemma 2.1, we have

$$
u(t)=\frac{1}{\Gamma(\beta)} \int_{0}^{t}(t-s)^{\beta-1} h(s) d s+C_{0}+C_{1} t+C_{2} t^{2}+\cdots+C_{m-1} t^{m-1} .
$$

Since $u^{(i)}(0)=0(i=1,2, \ldots, m-1)$, we have $C_{1}=C_{2}=\cdots=C_{m-1}=0$ and

$$
u(1)=\frac{1}{\Gamma(\beta)} \int_{0}^{1}(1-s)^{\beta-1} h(s) d s+C_{0} .
$$

Combined with $u(1)=0$, we know

$$
C_{0}=-\frac{1}{\Gamma(\beta)} \int_{0}^{1}(1-s)^{\beta-1} h(s) d s
$$

Thus

$$
\begin{aligned}
u(t)= & \frac{1}{\Gamma(\beta)} \int_{0}^{t}(t-s)^{\beta-1} h(s) d s-\frac{1}{\Gamma(\beta)} \int_{0}^{1}(1-s)^{\beta-1} h(s) d s \\
= & \frac{1}{\Gamma(\beta)} \int_{0}^{t}\left((t-s)^{\beta-1}-(1-s)^{\beta-1}\right) h(s) d s \\
& -\frac{1}{\Gamma(\beta)} \int_{t}^{1}(1-s)^{\beta-1} h(s) d s \\
= & -\int_{0}^{1} H(t, s) h(s) d s,
\end{aligned}
$$

where $H(t, s)$ is given by $(2.3)$. 
The proof is completed.

Lemma 2.4 Assume $\left(\mathrm{L}_{0}\right)$ holds, let $y \in C[0,1]$ and $1<n-1<\alpha<n, 1<m-1<\beta<m$. Then the following boundary value problems:

$$
\left\{\begin{array}{l}
D_{0^{+}}^{\alpha} x(t)=y(t), \quad 0<t<1, \\
x^{(j)}(0)=0, \quad j=2,3, \ldots, n-1, \\
x(1)=\int_{0}^{1} g_{1}(s) x(s) d s \\
x^{\prime}(0)=\int_{0}^{1} g_{2}(s) x(s) d s
\end{array}\right.
$$

has an unique solution

$$
x(t)=-\int_{0}^{1} G(t, s) y(s) d s
$$

and

$$
D_{0+}^{\beta} x(t)=\frac{1}{\Gamma(\alpha-\beta)} \int_{0}^{t}(t-s)^{\alpha-\beta-1} y(s) d s,
$$

where

$$
\begin{aligned}
& G(t, s)=G_{1}(t, s)+G_{2}(t, s), \\
& G_{1}(t, s)=\frac{1}{\Gamma(\alpha)} \begin{cases}(1-s)^{\alpha-1}-(t-s)^{\alpha-1}, & 0 \leq s \leq t \leq 1, \\
(1-s)^{\alpha-1}, & 0 \leq t \leq s \leq 1,\end{cases} \\
& G_{2}(t, s)=\delta\left(P(t) \int_{0}^{1} g_{1}(\tau) G_{1}(\tau, s) d \tau+Q(t) \int_{0}^{1} g_{2}(\tau) G_{1}(\tau, s) d \tau\right),
\end{aligned}
$$

here

$$
\begin{aligned}
& \delta^{-1}=\left(1-M_{1}\right)\left(1-N_{2}\right)+N_{1}\left(1-M_{2}\right), \\
& P(t)=1-N_{2}+N_{1} t, \quad Q(t)=M_{2}-1+\left(1-M_{1}\right) t, \\
& M_{1}=\int_{0}^{1} g_{1}(s) d s, \quad M_{2}=\int_{0}^{1} s g_{1}(s) d s, \\
& N_{1}=\int_{0}^{1} g_{2}(s) d s, \quad N_{2}=\int_{0}^{1} s g_{2}(s) d s .
\end{aligned}
$$

Proof From Lemma 2.1, considering BVP (2.6), we have

$$
x(t)=\frac{1}{\Gamma(\alpha)} \int_{0}^{t}(t-s)^{\alpha-1} y(s) d s+C_{0}+C_{1} t+C_{2} t^{2}+\cdots+C_{n-1} t^{n-1} .
$$

Because $x^{(j)}(0)=0(j=1,2, \ldots, n-1)$, we have $C_{2}=C_{3}=\cdots=C_{n-1}=0$, and

$$
x(1)=\frac{1}{\Gamma(\alpha)} \int_{0}^{1}(1-s)^{\alpha-1} y(s) d s+C_{0}+C_{1} .
$$


From the second condition of BVP (2.6), we have

$$
\int_{0}^{1} g_{1}(s) x(s) d s=\frac{1}{\Gamma(\alpha)} \int_{0}^{1}(1-s)^{\alpha-1} y(s) d s+C_{0}+C_{1}
$$

From the last condition of BVP (2.6), we have

$$
C_{1}=x^{\prime}(0)=\int_{0}^{1} g_{2}(s) x(s) d s
$$

By (2.16) and (2.17), we obtain

$$
C_{0}=\int_{0}^{1} g_{1}(s) x(s) d s-\int_{0}^{1} g_{2}(s) x(s) d s-\frac{1}{\Gamma(\alpha)} \int_{0}^{1}(1-s)^{\alpha-1} y(s) d s
$$

So

$$
\begin{aligned}
x(t)= & \frac{1}{\Gamma(\alpha)} \int_{0}^{t}(t-s)^{\alpha-1} y(s) d s+C_{0}+C_{1} t \\
= & \frac{1}{\Gamma(\alpha)} \int_{0}^{t}(t-s)^{\alpha-1} y(s) d s+\int_{0}^{1} g_{1}(s) x(s) d s-\int_{0}^{1} g_{2}(s) x(s) d s \\
& -\frac{1}{\Gamma(\alpha)} \int_{0}^{1}(1-s)^{\alpha-1} y(s) d s+t \int_{0}^{1} g_{2}(s) x(s) d s \\
= & \frac{1}{\Gamma(\alpha)} \int_{0}^{t}\left((t-s)^{\alpha-1}-(1-s)^{\alpha-1}\right) y(s) d s-\frac{1}{\Gamma(\alpha)} \int_{t}^{1}(1-s)^{\alpha-1} y(s) d s \\
& +\int_{0}^{1} g_{1}(s) x(s) d s-\int_{0}^{1} g_{2}(s) x(s) d s+t \int_{0}^{1} g_{2}(s) x(s) d s \\
= & -\int_{0}^{1} G_{1}(t, s) y(s) d s+A_{1}-A_{2}+t A_{2},
\end{aligned}
$$

where $G_{1}(t, s)$ is given in (2.10), and

$$
\begin{aligned}
& A_{1}=\int_{0}^{1} g_{1}(s) x(s) d s, \\
& A_{2}=\int_{0}^{1} g_{2}(s) x(s) d s .
\end{aligned}
$$

In view of (2.19), we get

$$
g_{1}(t) x(t)=-g_{1}(t) \int_{0}^{1} G_{1}(t, s) y(s) d s+A_{1} g_{1}(t)-A_{2} g_{1}(t)+t A_{2} g_{1}(t)
$$

Integrating (2.20) from 0 to 1 , we obtain

$$
\begin{aligned}
A_{1} & =\int_{0}^{1} g_{1}(s) x(s) d s \\
& =-\int_{0}^{1} g_{1}(s)\left(\int_{0}^{1} G_{1}(s, \tau) y(\tau) d \tau\right) d s
\end{aligned}
$$


Chang et al. Advances in Difference Equations ( 2017) 2017:36

Page 7 of 19

$$
\begin{aligned}
& +A_{1} \int_{0}^{1} g_{1}(s) d s-A_{2} \int_{0}^{1} g_{1}(s) d s+A_{2} \int_{0}^{1} s g_{1}(s) d s \\
= & I_{1}+A_{1} M_{1}-A_{2} M_{1}+A_{2} M_{2},
\end{aligned}
$$

where $M_{1}$ and $M_{2}$ are given in (2.14), and

$$
I_{1}=-\int_{0}^{1} g_{1}(s)\left(\int_{0}^{1} G_{1}(s, \tau) y(\tau) d \tau\right) d s=-\int_{0}^{1}\left(\int_{0}^{1} g_{1}(s) G_{1}(s, \tau) d s\right) y(\tau) d \tau .
$$

Similarly, we obtain

$$
\begin{aligned}
A_{2}= & \int_{0}^{1} g_{2}(s) x(s) d s \\
= & -\int_{0}^{1} g_{2}(s)\left(\int_{0}^{1} G_{1}(s, \tau) y(\tau) d \tau\right) d s \\
& +A_{1} \int_{0}^{1} g_{2}(s) d s-A_{2} \int_{0}^{1} g_{2}(s) d s+A_{2} \int_{0}^{1} s g_{2}(s) d s \\
= & I_{2}+A_{1} N_{1}-A_{2} N_{1}+A_{2} N_{2},
\end{aligned}
$$

where $N_{1}$ and $N_{2}$ are given in (2.15), and

$$
I_{2}=-\int_{0}^{1} g_{2}(s)\left(\int_{0}^{1} G_{1}(s, \tau) y(\tau) d \tau\right) d s=-\int_{0}^{1}\left(\int_{0}^{1} g_{2}(s) G_{1}(s, \tau) d s\right) y(\tau) d \tau .
$$

From (2.21) and (2.22), we get

$$
\begin{aligned}
& A_{1}=\left(I_{2}\left(M_{2}-M_{1}\right)+I_{1}\left(1+N_{1}-N_{2}\right)\right) \delta, \\
& A_{2}=\left(I_{2}\left(1-M_{1}\right)+I_{1} N_{1}\right) \delta,
\end{aligned}
$$

where $\delta^{-1}$ is given in (2.12). Hence,

$$
\begin{aligned}
x(t)= & -\int_{0}^{1} G_{1}(t, s) y(s) d s+A_{1}-A_{2}+t A_{2} \\
= & -\int_{0}^{1} G_{1}(t, s) y(s) d s+\delta\left(I_{2}\left(M_{2}-1+t-M_{1} t\right)+I_{1}\left(1-N_{2}+N_{1} t\right)\right) \\
= & -\int_{0}^{1} G_{1}(t, s) y(s) d s+\delta I_{1} P(t)+\delta I_{2} Q(t) \\
= & -\int_{0}^{1} G_{1}(t, s) y(s) d s-\int_{0}^{1} \delta P(t)\left(\int_{0}^{1} g_{1}(\tau) G_{1}(\tau, s) d \tau\right) y(s) d s \\
& -\int_{0}^{1} \delta Q(t)\left(\int_{0}^{1} g_{2}(\tau) G_{1}(\tau, s) d \tau\right) y(s) d s \\
= & -\int_{0}^{1} G_{1}(t, s) y(s) d s-\int_{0}^{1} \delta\left(P(t) \int_{0}^{1} g_{1}(\tau) G_{1}(\tau, s) d \tau\right. \\
& \left.+Q(t) \int_{0}^{1} g_{2}(\tau) G_{1}(\tau, s) d \tau\right) y(s) d s
\end{aligned}
$$




$$
\begin{aligned}
& =-\int_{0}^{1} G_{1}(t, s) y(s) d s-\int_{0}^{1} G_{2}(t, s) y(s) d s \\
& =-\int_{0}^{1} G(t, s) y(s) d s,
\end{aligned}
$$

where $G_{2}(t, s)$ is given in (2.11) and $P(t), Q(t)$ are given in (2.13).

On the other hand, in view of (2.19), because $m-1<\beta<\alpha-1<n-1$, by Lemma 2.2, we have

$$
\begin{aligned}
D_{0+}^{\beta} x(t) & =D_{0+}^{\beta}\left(\frac{1}{\Gamma(\alpha)} \int_{0}^{t}(t-s)^{\alpha-1} y(s) d s+C_{0}+C_{1} t\right) \\
& =D_{0+}^{\beta}\left(I_{0+}^{\alpha} y(t)+C_{0}+C_{1} t\right) \\
& =D_{0+}^{\beta} I_{0+}^{\alpha} y(t)+D_{0+}^{\beta}\left(C_{0}\right)+D_{0+}^{\beta}\left(C_{1} t\right) \\
& =D_{0+}^{\beta} I_{0+}^{\alpha} y(t) \\
& =I_{0+}^{\alpha-\beta} y(t) \\
& =\frac{1}{\Gamma(\alpha-\beta)} \int_{0}^{t}(t-s)^{\alpha-\beta-1} y(s) d s .
\end{aligned}
$$

The proof is completed.

Lemma 2.5 BVP (1.1) equivalent to the following integral equation:

$$
x(t)=\int_{0}^{1} G(t, s) \varphi_{q}\left(\int_{0}^{1} H(s, \tau) f\left(\tau, x(\tau), D_{0+}^{\beta} x(\tau)\right) d \tau\right) d s
$$

and

$$
D_{0+}^{\beta} x(t)=-\frac{1}{\Gamma(\alpha-\beta)} \int_{0}^{t}(t-s)^{\alpha-\beta-1} \varphi_{q}\left(\int_{0}^{1} H(s, \tau) f\left(\tau, x(\tau), D_{0+}^{\beta} x(\tau)\right) d \tau\right) d s,
$$

where $H(t, s)$ and $G(t, s)$ are given in (2.3) and (2.9).

Proof From Lemma 2.4 and Lemma 2.5, let $y(t)=\varphi_{q}(u(t)), h(t)=f\left(t, x(t), D_{0+}^{\beta} x(t)\right)$, we have

$$
\begin{aligned}
y(t) & =\varphi_{q}(u(t))=\varphi_{q}\left(-\int_{0}^{1} H(t, s) f\left(s, x(s), D_{0+}^{\beta} x(s)\right) d s\right) \\
& =-\varphi_{q}\left(\int_{0}^{1} H(t, s) f\left(s, x(s), D_{0+}^{\beta} x(s)\right) d s\right) .
\end{aligned}
$$

Immediately we obtain

$$
x(t)=-\int_{0}^{1} G(t, s) y(s) d s=\int_{0}^{1} G(t, s) \varphi_{q}\left(\int_{0}^{1} H(s, \tau) f\left(\tau, x(\tau), D_{0+}^{\beta} x(\tau)\right) d \tau\right) d s .
$$

From (2.23), we have (2.25).

The proof is completed.

Lemma 2.6 Assume $\left(\mathrm{L}_{0}\right)$ holds, then the function $H(t, s)$ defined by $(2.3)$ and the function $G(t, s)$ defined by (2.9) satisfies 
(1) $H(t, s) \geq 0$ is continuous for all $t, s \in[0,1]$;

(2) $H(t, s) \leq H(s, s)$ for all $t, s \in[0,1]$;

(3) $G(t, s) \geq 0$ is continuous for all $t, s \in[0,1]$.

Proof (1) From (2.3), it is easy to show that $H(t, s)$ is continuous on $[0,1] \times[0,1]$ and obviously, $H(t, s) \geq 0$, for $s \geq t$.

For $0 \leq s \leq t \leq 1$, we have

$$
(1-s)^{\beta-1}-(t-s)^{\beta-1}=(1-s)^{\beta-1}\left(1-\left(\frac{t-s}{1-s}\right)^{\beta-1}\right) \geq 0 .
$$

By (2.3), we know $H(t, s) \geq 0, t, s \in[0,1]$, and $H(t, s)>0, t, s \in(0,1)$.

(2) For $0 \leq s \leq t \leq 1$, we have

$$
H(t, s)=(1-s)^{\beta-1}-(t-s)^{\beta-1} \leq(1-s)^{\beta-1}=H(s, s)
$$

and for $s \geq t, H(t, s)=H(s, s)$, so that $H(t, s) \leq H(s, s)$, for all $t, s \in[0,1]$.

(3) From (2.9), we know $G(t, s)=G_{1}(t, s)+G_{2}(t, s)$. Firstly, from (2.10), similarly, we can obtain $G_{1}(t, s) \geq 0, t, s \in[0,1]$, and $G_{1}(t, s)>0, t, s \in(0,1)$. On the other hand, from $\left(\mathrm{L}_{0}\right)$, we know, for $t \in(0,1)$,

$$
g_{1}(t)>\operatorname{tg}_{1}(t)>\operatorname{tg}_{2}(t)
$$

and

$$
\begin{aligned}
& 1>\int_{0}^{1} g_{1}(s) d s>\int_{0}^{1} s g_{1}(s) d s>\int_{0}^{1} s g_{2}(s) d s>0, \\
& 1>\int_{0}^{1} g_{1}(s) d s>\int_{0}^{1} g_{2}(s) d s>\int_{0}^{1} s g_{2}(s) d s>0 .
\end{aligned}
$$

That implies

$$
1>M_{1}>M_{2}>N_{2}>0, \quad 1>M_{1}>N_{1}>N_{2}>0 .
$$

Therefore

$$
\delta^{-1}=\left(1-M_{1}\right)\left(1-N_{2}\right)+N_{1}\left(1-M_{2}\right)>0
$$

From $\left(\mathrm{L}_{0}\right)$ and $G_{1}(t, s) \geq 0$, we know

$$
\frac{\partial G_{2}(t, s)}{\partial t}=\delta\left(N_{1} \int_{0}^{1} g_{1}(\tau) G_{1}(\tau, s) d \tau+\left(1-M_{1}\right) \int_{0}^{1} g_{2}(\tau) G_{1}(\tau, s) d \tau\right)>0,
$$

which implies that $G_{2}(t, s)$ is a monotone increasing function with respect to $t \in[0,1]$.

So, from (2.11), we have

$$
\begin{aligned}
G_{2}(t, s) & \geq G_{2}(0, s)=\delta\left(P(0) \int_{0}^{1} g_{1}(\tau) G_{1}(\tau, s) d \tau+Q(0) \int_{0}^{1} g_{2}(\tau) G_{1}(\tau, s) d \tau\right) \\
& =\delta\left(\left(1-N_{2}\right) \int_{0}^{1} g_{1}(\tau) G_{1}(\tau, s) d \tau+\left(M_{2}-1\right) \int_{0}^{1} g_{2}(\tau) G_{1}(\tau, s) d \tau\right)
\end{aligned}
$$




$$
\begin{aligned}
& =\delta\left(\int_{0}^{1}\left(\left(1-N_{2}\right) g_{1}(\tau)+\left(M_{2}-1\right) g_{2}(\tau)\right) G_{1}(\tau, s) d \tau\right) \\
& \geq \delta\left(\int_{0}^{1}\left(\left(1-N_{2}\right) g_{2}(\tau)+\left(M_{2}-1\right) g_{2}(\tau)\right) G_{1}(\tau, s) d \tau\right) \\
& =\delta\left(M_{2}-N_{2}\right)\left(\int_{0}^{1} g_{2}(\tau) G_{1}(\tau, s) d \tau\right) \\
& \geq 0 .
\end{aligned}
$$

So $G_{2}(t, s) \geq 0$. Hence, $G(t, s) \geq 0$.

The proof is completed.

Lemma 2.7 Let $\eta \in\left(0, \frac{1}{2}\right)$, denote $I_{\eta}=[0, \eta]$ and $\rho_{1}=1-\left(\frac{\eta}{1-\eta}\right)^{\alpha-1}$, then

$$
\min _{t \in I_{\eta}} G_{1}(t, s) \geq \rho_{1} G_{1}(s, s)=\rho_{1} \max _{t \in[0,1]} G_{1}(t, s)
$$

Proof For $0 \leq s<t \leq 1$ and $t \in I_{\eta}$,

$$
G_{1}(t, s)=(1-s)^{\alpha-1}-(t-s)^{\alpha-1} \leq(1-s)^{\alpha-1}=G_{1}(s, s)
$$

and

$$
\begin{aligned}
\frac{G_{1}(t, s)}{G_{1}(s, s)} & =\frac{(1-s)^{\alpha-1}-(t-s)^{\alpha-1}}{(1-s)^{\alpha-1}} \\
& =1-\left(\frac{t-s}{1-s}\right)^{\alpha-1} \\
& \geq 1-\left(\frac{\eta}{1-s}\right)^{\alpha-1} \\
& \geq 1-\left(\frac{\eta}{1-\eta}\right)^{\alpha-1} \\
& =\rho_{1}>0 .
\end{aligned}
$$

For $s \geq t$ and $t \in I_{\eta}$,

$$
G_{1}(t, s)=G_{1}(s, s)>\left(1-\left(\frac{\eta}{1-\eta}\right)^{\alpha-1}\right) G_{1}(s, s)=\rho_{1} G_{1}(s, s) .
$$

Therefore,

$$
\min _{t \in I_{\eta}} G_{1}(t, s) \geq \rho_{1} G_{1}(s, s) .
$$

The proof is completed.

Lemma 2.8 Assume $\left(\mathrm{L}_{0}\right)$ holds, then the function $G_{2}(t, s)$ satisfies the following properties:

(1) $G_{2}(t, s) \leq G_{2}(1, s)=\max _{t \in[0,1]} G_{2}(t, s)$;

(2) $\min _{t \in I_{\eta}} G_{2}(t, s) \geq \rho_{2} \max _{t \in[0,1]} G_{2}(t, s)$, where $0<\rho_{2}=\frac{M_{2}-N_{2}}{1-N_{2}+N_{1}}<1$. 
Proof From Lemma 2.7 and (2.27), we obtain $G_{2}(t, s)>0$ and

$$
\begin{aligned}
\max _{t \in[0,1]} G_{2}(t, s) & =G_{2}(1, s) \\
& =\delta\left(\left(1-N_{2}+N_{1}\right) \int_{0}^{1} g_{1}(\tau) G_{1}(\tau, s) d \tau+\left(M_{2}-M_{1}\right) \int_{0}^{1} g_{2}(\tau) G_{1}(\tau, s) d \tau\right)
\end{aligned}
$$

and

$$
\begin{aligned}
\min _{t \in I_{\eta}} G_{2}(t, s) & =G_{2}(0, s) \\
& =\delta\left(\left(1-N_{2}\right) \int_{0}^{1} g_{1}(\tau) G_{1}(\tau, s) d \tau+\left(M_{2}-1\right) \int_{0}^{1} g_{2}(\tau) G_{1}(\tau, s) d \tau\right) \\
& \geq 0 .
\end{aligned}
$$

Furthermore,

$$
\begin{aligned}
\frac{G_{2}(0, s)}{G_{2}(1, s)} & =\frac{\left(1-N_{2}\right) \int_{0}^{1} g_{1}(\tau) G_{1}(\tau, s) d \tau+\left(M_{2}-1\right) \int_{0}^{1} g_{2}(\tau) G_{1}(\tau, s) d \tau}{\left(1-N_{2}+N_{1}\right) \int_{0}^{1} g_{1}(\tau) G_{1}(\tau, s) d \tau+\left(M_{2}-M_{1}\right) \int_{0}^{1} g_{2}(\tau) G_{1}(\tau, s) d \tau} \\
& >\frac{\left(1-N_{2}\right) \int_{0}^{1} g_{1}(\tau) G_{1}(\tau, s) d \tau+\left(M_{2}-1\right) \int_{0}^{1} g_{1}(\tau) G_{1}(\tau, s) d \tau}{\left(1-N_{2}+N_{1}\right) \int_{0}^{1} g_{1}(\tau) G_{1}(\tau, s) d \tau+\left(M_{2}-M_{1}\right) \int_{0}^{1} g_{2}(\tau) G_{1}(\tau, s) d \tau} \\
& >\frac{\left(1-N_{2}+M_{2}-1\right) \int_{0}^{1} g_{1}(\tau) G_{1}(\tau, s) d \tau}{\left(1-N_{2}+N_{1}\right) \int_{0}^{1} g_{1}(\tau) G_{1}(\tau, s) d \tau} \\
& =\frac{M_{2}-N_{2}}{1-N_{2}+N_{1}} \\
& =\rho_{2}
\end{aligned}
$$

and

$$
0<\rho_{2}=\frac{M_{2}-N_{2}}{1-N_{2}+N_{1}}<\frac{M_{2}-N_{2}}{1-N_{2}}<1 .
$$

Hence,

$$
\min _{t \in I_{\eta}} G_{2}(t, s) \geq \rho_{2} G_{2}(1, s)
$$

The proof is completed.

From Lemma 2.7 and Lemma 2.8, we can easily show that the following result holds.

\section{Lemma 2.9}

$$
\min _{t \in I_{\eta}} G(t, s) \geq \rho\left(G_{1}(s, s)+G_{2}(1, s)\right) \quad \text { and } \quad G(t, s) \leq G_{1}(s, s)+G_{2}(1, s),
$$

where $\rho=\min \left\{\rho_{1}, \rho_{2}\right\}$. 


\section{Main results}

In this section we deduce the existence of positive solutions to BVP (1.1) by using the wellknown Avery-Peterson fixed point theorem; see [4].

Let $\gamma$ and $\theta$ be non-negative continuous convex functionals on $P, \varphi$ be a non-negative continuous concave functional on $P$, and $\psi$ be a non-negative continuous functional on $P$. For $a, b, c, d>0$, we define the following convex set:

$$
\begin{aligned}
& P(\gamma ; d)=\{x \in P: \gamma(x)<d\}, \\
& P(\gamma, \varphi ; b, d)=\{x \in P: b \leq \varphi(x), \gamma(x) \leq d\}, \\
& P(\gamma, \theta, \varphi ; b, c, d)=\{x \in P: b \leq \varphi(x), \theta(x) \leq c, \gamma(x) \leq d\},
\end{aligned}
$$

and a closed set

$$
P(\gamma, \psi ; a, d)=\{x \in P: a \leq \psi(x), \gamma(x) \leq d\}
$$

Lemma 3.1 (see [4]) Let $P$ be a cone in a real Banach space E. Let $\gamma$ and $\theta$ be non-negative continuous convex functionals on $P, \varphi$ be a non-negative continuous concave functional on $P$, and $\psi$ be a non-negative continuous functional on $P$ satisfying $\psi(\lambda x) \leq \lambda \psi(x)$ for $0 \leq \lambda \leq 1$, such that, for some positive numbers $M$ and $d$,

$$
\varphi(x) \leq \psi(x), \quad\|x\| \leq M \gamma(x)
$$

for all $x \in \overline{P(\gamma ; d)}$. Suppose

$$
T: \overline{P(\gamma ; d)} \rightarrow \overline{P(\gamma ; d)}
$$

is completely continuous and there exist positive numbers $a, b$, and $c$ with $a<b$ such that

(H1) $\{x \in P(\gamma, \theta, \varphi ; b, c, d): \varphi(x)>b\} \neq \emptyset$, and $\varphi(x)>b$ for $x \in P(\gamma, \theta, \varphi ; b, c, d)$;

(H2) $\varphi(T x)>b$ for $x \in P(\gamma, \varphi ; b, d)$ with $\theta(T x)>c$;

(H3) $0 \notin P(\gamma, \psi ; a, d)$ and $\psi(T x)<a$ for $x \in P(\gamma, \psi ; a, d)$ with $\psi(x)=a$.

Then $T$ has at least three fixed point $x_{1}, x_{2}, x_{3} \in \overline{P(\gamma ; d)}$ such that

$$
\gamma\left(x_{i}\right) \leq d, \quad i=1,2,3 ; \quad \varphi\left(x_{1}\right)>b, \quad a<\varphi\left(x_{2}\right), \quad \psi\left(x_{2}\right)<b ; \quad \psi\left(x_{3}\right)<a .
$$

Let $E=\left\{x \in C[0,1]: D_{0+}^{\beta} x \in C[0,1], x^{\prime}(0)=\int_{0}^{1} g_{2}(s) x(s) d s, x^{(j)}(0)=0, j=2,3, \ldots, m-1\right\}$ be endowed with the norm

$$
\|x\|=\max \left\{\max _{t \in[0,1]}|x(t)|, \max _{t \in[0,1]}\left|D_{0+}^{\beta} x(t)\right|\right\}
$$

then $E$ is a Banach space.

We define a set $P \subset E$ by

$$
P=\left\{x \in E: x(t) \geq 0, D_{0+}^{\beta} x(t) \leq 0, \min _{t \in I_{\eta}} x(t) \geq \rho \max _{t \in[0,1]} x(t)\right\} .
$$


For $x, y \in P$ and $k_{1}, k_{2} \geq 0$, it is easy to obtain

$$
k_{1} x(t)+k_{2} y(t) \geq 0, \quad D_{0+}^{\beta}\left(k_{1} x(t)+k_{2} y(t)\right)=k_{1} D_{0+}^{\beta} x(t)+k_{2} D_{0+}^{\beta} y(t) \leq 0
$$

and

$$
\begin{aligned}
\min _{t \in I_{\eta}}\left\{k_{1} x(t)+k_{2} y(t)\right\} & \geq \min _{t \in I_{\eta}}\left\{k_{1} x(t)\right\}+\min _{t \in I_{\eta}}\left\{k_{2} y(t)\right\} \\
& \geq \rho \max _{t \in[0,1]}\left\{k_{1} x(t)\right\}+\rho \max _{t \in[0,1]}\left\{k_{2} y(t)\right\} \\
& =\rho\left(\max _{t \in[0,1]}\left\{k_{1} x(t)\right\}+\max _{t \in[0,1]}\left\{k_{2} y(t)\right\}\right) \\
& \geq \rho \max _{t \in[0,1]}\left\{k_{1} x(t)+k_{2} y(t)\right\} .
\end{aligned}
$$

Thus, for $x, y \in P$ and $k_{1}, k_{2} \geq 0, k_{1} x(t)+k_{2} y(t) \in P$. And if $x \in P, x \neq 0$, it is easy to prove that $-x \notin P$. Therefore, $P$ is a cone in $E$.

Let $T: P \rightarrow E$ be the operator defined by

$$
T x(t):=\int_{0}^{1} G(t, s) \varphi_{q}\left(\int_{0}^{1} H(s, \tau) f\left(\tau, x(\tau), D_{0+}^{\beta} x(\tau)\right) d \tau\right) d s .
$$

Then we have the following conclusion.

Lemma 3.2 Assume $\left(\mathrm{L}_{0}\right)$ holds, then $T: P \rightarrow P$ is a completely continuous operator.

Proof For $x \in P$, from the non-negativity and continuity of $G(t, s), H(t, s), f\left(t, x(t), D_{0+}^{\beta} x(t)\right)$, we know that $T$ is a continuous operator and $T x(t) \geq 0$. By (2.25), we have

$$
D_{0+}^{\beta} T x(t)=-\frac{1}{\Gamma(\alpha-\beta)} \int_{0}^{t}(t-s)^{\alpha-\beta-1} \varphi_{q}\left(\int_{0}^{1} H(s, \tau) f\left(\tau, x(\tau), D_{0+}^{\beta} x(\tau)\right) d \tau\right) d s \leq 0 .
$$

Furthermore,

$$
\begin{aligned}
\min _{t \in I_{\eta}} T x(t) & =\min _{t \in I_{\eta}} \int_{0}^{1} G(t, s) \varphi_{q}\left(\int_{0}^{1} H(s, \tau) f\left(\tau, x(\tau), D_{0+}^{\beta} x(\tau)\right) d \tau\right) d s \\
& =\int_{0}^{1} \min _{t \in I_{\eta}} G(t, s) \varphi_{q}\left(\int_{0}^{1} H(s, \tau) f\left(\tau, x(\tau), D_{0+}^{\beta} x(\tau)\right) d \tau\right) d s \\
& \geq \int_{0}^{1} \rho \max _{t \in[0,1]} G(t, s) \varphi_{q}\left(\int_{0}^{1} H(s, \tau) f\left(\tau, x(\tau), D_{0+}^{\beta} x(\tau)\right) d \tau\right) d s=\rho \max _{t \in[0,1]} T x(t) .
\end{aligned}
$$

Thus, $T(P) \subseteq P$.

Next, we show that $T$ is uniformly bounded.

Let $D \subset P$ be bounded, i.e., there exists a positive constant $r$ such that $\|x\| \leq r$, for all $x \in D$. Let $M_{0}=\max _{t \in[0,1], x \in D}\left|f\left(t, x(t), D_{0+}^{\beta} x(t)\right)\right|+1>0$, for $x \in D$, we have

$$
\begin{aligned}
|T x(t)| & =\left|\int_{0}^{1} G(t, s) \varphi_{q}\left(\int_{0}^{1} H(s, \tau) f\left(\tau, x(\tau), D_{0+}^{\beta} x(\tau)\right) d \tau\right) d s\right| \\
& \leq \int_{0}^{1}|G(t, s)| \varphi_{q}\left(\int_{0}^{1}|H(s, \tau)|\left|f\left(\tau, x(\tau), D_{0+}^{\beta} x(\tau)\right)\right| d \tau\right) d s
\end{aligned}
$$




$$
\begin{aligned}
& \leq \varphi_{q}\left(M_{0}\right) \int_{0}^{1} G(t, s) \varphi_{q}\left(\int_{0}^{1} H(s, \tau)\right) d s \\
& \leq \varphi_{q}\left(M_{0}\right) \int_{0}^{1}\left(G_{1}(s, s)+G_{2}(1, s)\right) \varphi_{q}\left(\int_{0}^{1} H(s, \tau)\right) d s:=M_{01} .
\end{aligned}
$$

Furthermore, for any $x \in D$ and $t \in[0,1]$, we have

$$
\begin{aligned}
\left|D_{0+}^{\beta} T x(t)\right| & =\left|-\frac{1}{\Gamma(\alpha-\beta)} \int_{0}^{t}(t-s)^{\alpha-\beta-1} \varphi_{q}\left(\int_{0}^{1} H(s, \tau) f\left(\tau, x(\tau), D_{0+}^{\beta} x(\tau)\right) d \tau\right) d s\right| \\
& \leq \frac{1}{\Gamma(\alpha-\beta)} \int_{0}^{t}(t-s)^{\alpha-\beta-1} \varphi_{q}\left(\int_{0}^{1} H(s, \tau) f\left(\tau, x(\tau), D_{0+}^{\beta} x(\tau)\right) d \tau\right) d s \\
& \leq \varphi_{q}\left(M_{0}\right) \frac{1}{\Gamma(\alpha-\beta)} \int_{0}^{1}(1-s)^{\alpha-\beta-1} \varphi_{q}\left(\int_{0}^{1} H(s, \tau) d \tau\right) d s:=M_{02} .
\end{aligned}
$$

So $\|T x\| \leq \max \left\{M_{01}, M_{02}\right\}$, which implies $T$ is uniformly bounded.

In the following of the proof, we will prove that $T$ is equicontinuous. Since $G(t, s)$ is continuous on $[0,1] \times[0,1]$, it is uniformly continuous on $[0,1] \times[0,1]$. Thus, for any $\epsilon>0$, there exists a constant $\delta_{1}>0$, such that

$$
\left|G\left(t_{1}, s\right)-G\left(t_{2}, s\right)\right|<\frac{\epsilon}{\varphi_{q}\left(M_{0}\right) \int_{0}^{1} \varphi_{q}\left(\int_{0}^{1} H(s, \tau) d \tau\right) d s}
$$

for $t_{1}, t_{2} \in[0,1]$ with $\left|t_{1}, t_{2}\right|<\delta_{1}$. Therefore,

$$
\begin{aligned}
\left|T x\left(t_{1}\right)-T x\left(t_{2}\right)\right|= & \mid \int_{0}^{1} G\left(t_{1}, s\right) \varphi_{q}\left[\int_{0}^{1} H(s, \tau) f\left(\tau, x(\tau), D_{0+}^{\beta} x(\tau)\right) d \tau\right] d s \\
& -\int_{0}^{1} G\left(t_{2}, s\right) \varphi_{q}\left(\int_{0}^{1} H(s, \tau) f\left(\tau, x(\tau), D_{0+}^{\beta} x(\tau)\right) d \tau\right) d s \mid \\
\leq & \int_{0}^{1}\left|G\left(t_{1}, s\right)-G\left(t_{2}, s\right)\right| \varphi_{q}\left(\int_{0}^{1} H(s, \tau) f\left(\tau, x(\tau), D_{0+}^{\beta} x(\tau)\right) d \tau\right) d s \\
& <\frac{\epsilon}{\varphi_{q}\left(M_{0}\right) \int_{0}^{1} \varphi_{q}\left(\int_{0}^{1} H(s, \tau) d \tau\right) d s} \varphi_{q}\left(M_{0}\right) \int_{0}^{1} \varphi_{q}\left(\int_{0}^{1} H(s, \tau) d \tau\right) d s \\
= & \epsilon .
\end{aligned}
$$

On the other hand, for $1<m-1<\beta<\alpha-1<n-1, t^{\alpha-\beta}$ is uniformly continuous on $[0,1]$. We denote $M_{03}=\frac{\varphi_{q}\left(M_{0}\right)}{(\alpha-\beta) \Gamma(\alpha-\beta)}$. Then there exists a constant $0<\delta_{2}<\left(\frac{\epsilon}{4 M_{03}}\right)^{(\alpha-\beta)^{-1}}$, such that, for any $0<t_{1}<t_{2}<1$ and $\left|t_{2}-t_{1}\right|<\delta_{2}$, we have

$$
\left|t_{2}^{\alpha-\beta}-t_{1}^{\alpha-\beta}\right|<\frac{\epsilon}{2 M_{03}} .
$$

Thus, from Lemma 2.7, we have

$$
\begin{aligned}
\left|D_{0+}^{\beta} T x\left(t_{2}\right)-D_{0+}^{\beta} T x\left(t_{1}\right)\right| \\
=\mid \frac{1}{\Gamma(\alpha-\beta)} \int_{0}^{t_{2}}\left(t_{2}-s\right)^{\alpha-\beta-1} \varphi_{q}\left(\int_{0}^{1} H(s, \tau) f\left(\tau, x(\tau), D_{0+}^{\beta} x(\tau)\right) d \tau\right) d s \\
\quad-\frac{1}{\Gamma(\alpha-\beta)} \int_{0}^{t_{1}}\left(t_{1}-s\right)^{\alpha-\beta-1} \varphi_{q}\left(\int_{0}^{1} H(s, \tau) f\left(\tau, x(\tau), D_{0+}^{\beta} x(\tau)\right) d \tau\right) d s \mid
\end{aligned}
$$




$$
\begin{aligned}
\leq & \frac{1}{\Gamma(\alpha-\beta)}\left(\mid \int_{0}^{t_{1}}\left(\left(t_{2}-s\right)^{\alpha-\beta-1}-\left(t_{1}-s\right)^{\alpha-\beta-1}\right)\right. \\
& \times \varphi_{q}\left(\int_{0}^{1} H(s, \tau) f\left(\tau, x(\tau), D_{0+}^{\beta} x(\tau)\right) d \tau\right) d s \mid \\
& \left.+\left|\int_{t_{1}}^{t_{2}}\left(t_{2}-s\right)^{\alpha-\beta-1} \varphi_{q}\left(\int_{0}^{1} H(s, \tau) f\left(\tau, x(\tau), D_{0+}^{\beta} x(\tau)\right) d \tau\right) d s\right|\right) \\
\leq & \frac{\varphi_{q}\left(\int_{0}^{1} M_{0} H(\tau, \tau) d \tau\right)}{\Gamma(\alpha-\beta)}\left(\left|\int_{0}^{t_{1}}\left(\left(t_{2}-s\right)^{\alpha-\beta-1}-\left(t_{1}-s\right)^{\alpha-\beta-1}\right) d s\right|\right. \\
& \left.+\left|\int_{t_{1}}^{t_{2}}\left(t_{2}-s\right)^{\alpha-\beta-1} d s\right|\right) \\
= & M_{03}\left(\left|\int_{0}^{t_{1}} d\left[\left(t_{1}-s\right)^{\alpha-\beta}-\left(t_{2}-s\right)^{\alpha-\beta}\right]\right|+\left|\int_{t_{1}}^{t_{2}} d\left(t_{2}-s\right)^{\alpha-\beta}\right|\right) \\
= & M_{03}\left(\left|0-\left(t_{2}-t_{1}\right)^{\alpha-\beta}-t_{1}^{\alpha-\beta}+t_{2}^{\alpha-\beta}\right|+\left|\left(t_{2}-t_{1}\right)^{\alpha-\beta}\right|\right) \\
\leq & M_{03}\left(\left|2\left(t_{2}-t_{1}\right)^{\alpha-\beta}\right|+\left|t_{2}^{\alpha-\beta}-t_{1}^{\alpha-\beta}\right|\right) \\
< & M_{03}\left(2\left(\left(\frac{\epsilon}{4 M_{03}}\right)^{(\alpha-\beta)^{-1}}\right)^{\alpha-\beta}+\frac{\epsilon}{2 M_{03}}\right) \\
= & .
\end{aligned}
$$

Hence, $T$ is equicontinuous.

According to the Arzelà-Ascoli theorem, $T$ is a completely continuous operator. The proof is completed.

Denote the positive constants

$$
\begin{aligned}
& J_{1}=\int_{0}^{1}\left(G_{1}(s, s)+G_{2}(1, s)\right) \varphi_{q}\left(\int_{0}^{1} H(s, \tau) d \tau\right) d s, \\
& J_{2}=\frac{1}{\Gamma(\alpha-\beta)} \int_{0}^{1}(1-s)^{\alpha-\beta-1} \varphi_{q}\left(\int_{0}^{1} H(s, \tau) d \tau\right) d s,
\end{aligned}
$$

and

$$
J_{3}=\int_{0}^{1}\left(G_{1}(s, s)+G_{2}(1, s)\right) \varphi_{q}\left(\int_{0}^{\eta} H(s, \tau) d \tau\right) d s
$$

Define the functionals as follows:

$$
\gamma(x)=\|x\|, \quad \theta(x)=\psi(x)=\max _{t \in[0,1]}|x(t)|, \quad \varphi(x)=\min _{t \in I_{\eta}}|x(t)|,
$$

then $\gamma$ and $\theta$ are continuous non-negative convex functionals, $\varphi$ is a continuous nonnegative concave functional, $\psi$ is a continuous non-negative functional, and

$$
\rho \theta(x) \leq \varphi(x) \leq \theta(x)=\psi(x), \quad\|x\| \leq M \gamma(x),
$$

where $M=1$. Therefore, condition (3.1) in Lemma 3.1 is satisfied. 
Theorem 3.1 Suppose $\left(\mathrm{L}_{0}\right)$ holds, and there exist constants $0<a, b, d$ with $a<b<$ $\rho d \min \left\{\frac{J_{3}}{J_{1}}, \frac{J_{3}}{J_{2}}\right\}$ and $c=\frac{b}{\rho}$, such that

(L $\left.\mathrm{L}_{1}\right) f(t, x, y) \leq \min \left\{\varphi_{p}\left(\frac{d}{J_{1}}\right), \varphi_{p}\left(\frac{d}{J_{2}}\right)\right\},(t, x, y) \in[0,1] \times[0, d] \times[-d, 0]$;

$\left(\mathrm{L}_{2}\right) f(t, x, y)>\varphi_{p}\left(\frac{b}{\rho J_{3}}\right),(t, x, y) \in[0, \eta] \times\left[b, \frac{b}{\rho}\right] \times[-d, 0]$;

(L $\left.\mathrm{L}_{3}\right) f(t, x, y)<\varphi_{p}\left(\frac{a}{J_{1}}\right),(t, x, y) \in[0,1] \times[0, a] \times[-d, 0]$.

Then BVP (1.1) has at least three positive solutions $x_{1}, x_{2}, x_{3}$, satisfying

$$
\begin{aligned}
& \left\|x_{i}\right\| \leq d \quad(i=1,2,3), \\
& \min _{t \in I_{\eta}}\left|x_{1}(t)\right|>b, \quad a<\min _{t \in I_{\eta}}\left|x_{2}(t)\right|, \quad \max _{t \in[0,1]}\left|x_{2}(t)\right|<b, \quad \max _{t \in[0,1]}\left|x_{3}(t)\right|<a .
\end{aligned}
$$

Proof It is clear that the fixed points of operator $T$ are equivalent the solutions of BVP (1.1). For $x \in \overline{P(\gamma ; d)}$, we have

$$
\gamma(x)=\|x\| \leq d,
$$

this implies

$$
\max _{t \in[0,1]}|x(t)| \leq d, \quad \max _{t \in[0,1]}\left|D_{0+}^{\beta} x(t)\right| \leq d,
$$

then

$$
0 \leq x(t) \leq d, \quad-d \leq D_{0+}^{\beta} x(t) \leq 0 .
$$

By $\left(\mathrm{L}_{1}\right)$, we have

$$
\begin{aligned}
\max _{t \in[0,1]}|T x(t)| & =\max _{t \in[0,1]} \int_{0}^{1} G(t, s) \varphi_{q}\left(\int_{0}^{1} H(s, \tau) f\left(\tau, x(\tau), D_{0+}^{\beta} x(\tau)\right) d \tau\right) d s \\
& \leq \int_{0}^{1}\left(G_{1}(s, s)+G_{2}(1, s)\right) \varphi_{q}\left(\int_{0}^{1} H(s, \tau) f\left(\tau, x(\tau), D_{0+}^{\beta} x(\tau)\right) d \tau\right) d s \\
& \leq \int_{0}^{1}\left(G_{1}(s, s)+G_{2}(1, s)\right) \varphi_{q}\left(\varphi_{p}\left(\frac{d}{J_{1}}\right) \int_{0}^{1} H(s, \tau) d \tau\right) d s \\
& =\frac{d}{J_{1}} \int_{0}^{1}\left(G_{1}(s, s)+G_{2}(1, s)\right) \varphi_{q}\left(\int_{0}^{1} H(s, \tau) d \tau\right) d s \\
& =d
\end{aligned}
$$

and

$$
\begin{aligned}
\max _{t \in[0,1]}\left|D_{0+}^{\beta} T x(t)\right|= & \max _{t \in[0,1]} \mid \frac{-1}{\Gamma(\alpha-\beta)} \int_{0}^{t}(t-s)^{\alpha-\beta-1} \\
& \times \varphi_{q}\left(\int_{0}^{1} H(s, \tau) f\left(\tau, x(\tau), D_{0+}^{\beta} x(\tau)\right) d \tau\right) d s \mid \\
\leq & \frac{1}{\Gamma(\alpha-\beta)} \int_{0}^{1}(1-s)^{\alpha-\beta-1} \varphi_{q}\left(\varphi_{p}\left(\frac{d}{J_{2}}\right) \int_{0}^{1} H(s, \tau) d \tau\right) d s
\end{aligned}
$$




$$
\begin{aligned}
& =\frac{d}{J_{2}} \frac{1}{\Gamma(\alpha-\beta)} \int_{0}^{1}(1-s)^{\alpha-\beta-1} \varphi_{q}\left(\int_{0}^{1} H(s, \tau) d \tau\right) d s \\
& =d
\end{aligned}
$$

so

$$
\gamma(T x)=\|T x\|=\max \left\{\max _{t \in[0,1]}|T x(t)|, \max _{t \in[0,1]}\left|D_{0+}^{\beta} T x(t)\right|\right\} \leq d .
$$

Therefore $T: \overline{P(\gamma ; d)} \rightarrow \overline{P(\gamma ; d)}$.

Let $x(t)=\frac{b}{\rho}, x(t) \in P(\gamma, \theta, \varphi ; b, c, d)$ and $\varphi\left(\frac{b}{\rho}\right)>b$, which implies that

$$
\{x \in P(\gamma, \theta, \varphi ; b, c, d): \varphi(x)>b\} \neq \varnothing .
$$

For $x \in P(\gamma, \theta, \varphi ; b, c, d)$, we know that $b \leq x(t) \leq c=\frac{b}{\rho}$ for $t \in I_{\eta}$ and $-d \leq D_{0+}^{\beta} x(t) \leq 0$. In view of $\left(\mathrm{L}_{2}\right)$,

$$
\begin{aligned}
\varphi(T x) & =\min _{t \in I_{\eta}}|T x(t)| \\
& =\min _{t \in I_{\eta}} \int_{0}^{1} G(t, s) \varphi_{q}\left(\int_{0}^{1} H(s, \tau) f\left(\tau, x(\tau), D_{0+}^{\beta} x(\tau)\right) d \tau\right) d s \\
& >\int_{0}^{1} \rho\left(G_{1}(s, s)+G_{2}(1, s)\right) \varphi_{q}\left(\int_{0}^{\eta} H(s, \tau) \varphi_{p}\left(\frac{b}{\rho J_{3}}\right) d \tau\right) d s \\
& =\rho \frac{b}{\rho J_{3}} \int_{0}^{1}\left(G_{1}(s, s)+G_{2}(1, s)\right) \varphi_{q}\left(\int_{0}^{\eta} H(s, \tau) d \tau\right) d s \\
& =b .
\end{aligned}
$$

So $\varphi(T x)>b$ for all $x \in P(\gamma, \theta, \varphi ; b, c, d)$. Hence, the condition (H1) of Lemma 3.1 is satisfied.

By (3.2), for all $x \in P(\gamma, \varphi ; b, d)$ with $\theta(T x)>c=\frac{b}{\rho}$, we have

$$
\varphi(T x) \geq \rho \theta(T x)>\rho c=\rho \frac{b}{\rho}=b .
$$

Thus, the condition (H2) of Lemma 3.1 holds.

Because of $\psi(0)=0<a$, then $0 \notin P(\gamma, \psi ; a, d)$. For $x \in P(\gamma, \psi ; a, d)$ with $\psi(x)=a$, we know $\gamma(x) \leq d$. It means that $\max _{t \in[0,1]} x(t)=a$ and $-d \leq D_{0+}^{\beta} x(t) \leq 0$.

From $\left(\mathrm{L}_{3}\right)$, we can obtain

$$
\begin{aligned}
\psi(T x) & =\max _{t \in[0,1]}|T x(t)| \\
& =\max _{t \in[0,1]} \int_{0}^{1} G(t, s) \varphi_{q}\left(\int_{0}^{1} H(s, \tau) f\left(\tau, x(\tau), D_{0+}^{\beta} x(\tau)\right) d \tau\right) d s \\
& <\int_{0}^{1}\left(G_{1}(s, s)+G_{2}(1, s)\right) \varphi_{q}\left(\int_{0}^{1} H(s, \tau) \varphi_{p}\left(\frac{a}{J_{1}}\right) d \tau\right) d s \\
& =\frac{a}{J_{1}} \int_{0}^{1}\left(G_{1}(s, s)+G_{2}(1, s)\right) \varphi_{q}\left(\int_{0}^{1} H(s, \tau) d \tau\right) d s \\
& =a .
\end{aligned}
$$

Therefore, the condition (H3) of Lemma 3.1 holds. 
To sum up, the conditions of Lemma 3.1 are all verified. Hence, BVP (1.1) has at least three positive solutions $x_{1}, x_{2}, x_{3}$ satisfying (3.2) and (3.3).

The proof is completed.

\section{Example}

In this section, we present an example to illustrate the main result. Consider the following boundary value problems:

$$
\left\{\begin{array}{l}
D_{0+}^{\frac{5}{4}} \varphi_{\frac{3}{2}}\left(D_{0+}^{\frac{5}{2}} x(t)\right)=f\left(t, x(t), D_{0+}^{\frac{5}{4}} x(t)\right), \quad t \in(0,1) \\
\left(\varphi_{\frac{3}{2}}\left(D_{0+}^{\frac{5}{2}} x(0)\right)\right)^{\prime}=\varphi_{\frac{3}{2}}\left(D_{0+}^{\frac{5}{2}} x(1)\right)=0 \\
x(1)=\int_{0}^{1} s x(s) d s \\
x^{\prime}(0)=\int_{0}^{1} s^{2} x(s) d s \\
x^{\prime \prime}(0)=0
\end{array}\right.
$$

where $\alpha=\frac{5}{2}, \beta=\frac{5}{4}, p=\frac{3}{2}, g_{1}(t)=t, g_{2}(t)=t^{2}$, and

$$
f(t, x, y)= \begin{cases}\tan (0.6 t)+0.01 e^{x / 5} x^{2}+\sin (y), & 0 \leq x \leq 18 \\ \tan (0.6 t)+\sin (y)+92, & 18<x \leq 5,000\end{cases}
$$

Choose $a=8, b=18, d=5,000, \eta=\frac{1}{3}$. By a simple computation, we have

$$
\begin{aligned}
& \rho_{1}=\frac{1}{2}, \quad \rho_{2}=\frac{1}{13}, \quad \rho=\frac{1}{13}, \quad r=1, \\
& M_{1}=\frac{1}{2}, \quad M_{2}=\frac{1}{3}, \quad N_{1}=\frac{1}{3}, \quad N_{2}=\frac{1}{4}, \quad \delta=\frac{72}{43}, \\
& J_{1}=0.26227, \quad J_{2}=0.312484, \quad J_{3}=0.0286711 .
\end{aligned}
$$

We can check that the nonlinear term $f(t, x, y)$ satisfies

$\left(\mathrm{L}_{1}\right) f(t, x, y) \leq \min \left\{\varphi_{p}\left(\frac{d}{J_{1}}\right), \varphi_{p}\left(\frac{d}{J_{2}}\right)\right\} \approx 126.494,(t, x, y) \in[0,1] \times[0,5,000] \times[-5,000,0] ;$

$\left(\mathrm{L}_{2}\right) f(t, x, y)>\varphi_{p}\left(\frac{b}{\rho J_{3}}\right) \approx 90.3412,(t, x, y) \in\left[0, \frac{1}{3}\right] \times[18,234] \times[-5,000,0]$;

$\left(\mathrm{L}_{3}\right) f(t, x, y)<\varphi_{p}\left(\frac{a}{J_{1}}\right) \approx 5.52294,(t, x, y) \in[0,1] \times[0,8] \times[-5,000,0]$.

Then all assumptions of Theorem 3.1 are satisfied. Thus, BVP (4.1) has at least three positive solutions $x_{1}, x_{2}, x_{3}$, satisfying

$$
\begin{aligned}
& \left\|x_{i}\right\| \leq 5,000 \quad(i=1,2,3), \\
& \min _{t \in I_{\eta}}\left|x_{1}\right|>18, \quad 8<\min _{t \in I_{\eta}}\left|x_{2}\right|, \quad \max _{t \in[0,1]}\left|x_{2}\right|<18, \quad \max _{t \in[0,1]}\left|x_{3}\right|<8 .
\end{aligned}
$$




\section{Acknowledgements}

The author would like to thank the anonymous referees and the editor for their constructive suggestions on improving the presentation of the paper. Also, this research was supported by National Natural Science Foundation of China (Grant No. 11471215)

Received: 7 November 2016 Accepted: 14 January 2017 Published online: 01 February 2017

\section{References}

1. Oldham, KB, Spanier, J: The Fractional Calculus: Theory and Applications of Differentiation and Integration to Arbitrary Order. Mathematics in Science and Engineering. Academic Press, New York (1974)

2. Samko, SG, Kilbas, AA, Marichev, Ol: Fractional Integral and Derivatives: Theory and Applications. Gordon \& Breach, Yverdon (1993)

3. Podlubny, I: Fractional Differential Equations. Academic Press, New York (1999)

4. Avery, Rl, Peteson, AC: Three positive fixed points of nonlinear operators on ordered Banach spaces. Comput. Math. Appl. 42, 313-322 (2001)

5. Agrawal, OP: Formulation of Euler-Lagrange equations for fractional variational problems. J. Math. Anal. Appl. 272, 368-379 (2002)

6. Weitzner, H, Zaslavsky, GM: Some applications of fractional equations. Commun. Nonlinear Sci. Numer. Simul. 8, 273-281 (2003)

7. Bai, Z, Lu, H: Positive solutions for boundary value problem of nonlinear fractional differential equation. J. Math. Anal. Appl. 311(2), 495-505 (2005)

8. Kilbas, AA, Srivastava, HM, Trujillo, JJ: Theory and Applications of Fractional Differential Equations. Elsevier, New York (2006)

9. Meral, FC, Royston, TJ, Magin, R: Fractional calculus in viscoelasticity: an experimental study. Commun. Nonlinear Sci. Numer. Simul. 15, 939-945 (2010)

10. Machado, JT, Kiryakova, V, Mainardi, F: Recent history of fractional calculus. Commun. Nonlinear Sci. Numer. Simul. 16 1140-1153 (2011)

11. Liu, X, Jia, M, Xiang, X: On the solvability of a fractional differential equation model involving the $p$-Laplacian operator. Comput. Math. Appl. 64, 3267-3275 (2012)

12. Lu, H, Han, Z, Sun, S, Liu, J: Existence on positive solutions for boundary value problems of nonlinear fractional differential equations with p-Laplacian. Adv. Differ. Equ. 2012, Article ID 30 (2012)

13. Ahmad, B: Nonlinear fractional differential equations with anti-periodic type fractional boundary conditions. Differ. Equ. Dyn. Syst. 21(4), 387-401 (2013)

14. Zhang, $X$, Liu, L, Wiwatanapataphee, $B, W u, Y$ : The eigenvalue for a class of singular $p$-Laplacian fractional differential equations involving the Riemann-Stieltjes integral boundary condition. Appl. Math. Comput. 235, 412-422 (2014)

15. Su, Y, Li, $Q, L i u, X$ : Existence criteria for positive solutions of $p$-Laplacian fractional differential equations with derivative terms. Adv. Differ. Equ. 2013, Article ID 119 (2013)

16. Liu, X, Jia, M, Ge, W: Multiple solutions of a $p$-Laplacian model involving a fractional derivative. Adv. Differ. Equ. 2013 Article ID 126 (2013)

17. Liang, S, Zhang, J: Existence and uniqueness of positive solutions for integral boundary problems of nonlinear fractional differential equations with $p$-Laplacian operator. Rocky Mt. J. Math. 44(3), 953-974 (2014)

18. Liu, X, Jia, M, Ge, W: The method of lower and upper solutions for mixed fractional four-point boundary value problem whit $p$-Laplacian operator. Appl. Math. Lett. 65, 56-62 (2017)

19. Lomtatidze, A, Malaguti, L: On a nonlocal boundary value problems for second order nonlinear singular differential equations. Georgian Math. J. 7, 133-154 (2000)

20. Gallardo, JM: Second order differential operators with integral boundary conditions and generation of semigroups. Rocky Mt. J. Math. 30, 1265-1292 (2000)

21. Zhang, $X$, Feng, $M, G e, W$ : Symmetric positive solutions for $p$-Laplacian fourth-order differential equations with integral boundary conditions. J. Comput. Appl. Math. 222, 561-573 (2008)

22. $\mathrm{Ma}, \mathrm{R}, \mathrm{Xu}, \mathrm{L}$ : Existence of positive solutions of nonlinear fourth-order boundary value problem. Appl. Math. Lett. 23, $537-543(2010)$

23. Jia, M, Liu, X: Multiplicity of solutions for integral boundary value problems of fractional differential equations with upper and lower solutions. Appl. Math. Comput. 232, 313-323 (2014)

24. Zhi, E, Liu, X, Li, F: Nonlocal boundary value problem for fractional differential equations with $p$-Laplacian. Math. Methods Appl. Sci. 37, 2651-2662 (2014)

25. Senlik, T, Hamal, NA: Existence and multiplicity of symmetric positive solutions for nonlinear boundary-value problems with p-Laplacian operator. Bound. Value Probl. 2014, Article ID 44 (2014)

26. Mahmudov, $\mathrm{NI}$, Unul, S: Existence of solutions of fractional boundary value problems with $p$-Laplacian. Bound. Value Probl. 2015, Article ID 99 (2015) 